\title{
Classical Physics-based Renewable and Sustainable Energy Transition Concept
}

\author{
Anatoly Blanovsky \\ Westside Environmental Technology Company, Los Angeles, the United States
}

\section{Email address:}

ablanovsky@yahoo.com

\section{To cite this article:}

Anatoly Blanovsky. Classical Physics-based Renewable and Sustainable Energy Transition Concept. American Journal of Modern Physics. Vol. 10, No. 4, 2021, pp. 93-100. doi: 10.11648/j.ajmp.20211004.13

Received: June 9, 2021; Accepted: July 1, 2021; Published: August 31, 2021

\begin{abstract}
By many accounts, Lorentz was ahead of his time by suggesting before quantum mechanics that some disturbances, like waves, travel with particles through a vacuum without moving it. The waves associated with the Lorentz metrics are described by the Klein-Gordon equation and dispersion relation $\omega^{2}=c^{2} k^{2}+\omega_{c}^{2}$. In hydromechanics, they are known as non-propagating waves and $\omega_{\mathrm{c}}$ is a constant called the cutoff frequency. The waves are not propagating if their frequency is below the cutoff frequency $\omega_{\mathrm{c}}$. Their group and phase velocity are related by $\mathrm{vu}=\mathrm{c}^{2}$, in the infinite $\mathrm{k}$ limit $\mathrm{v}=\mathrm{u}=\mathrm{c}$. A quantum object is considered as a particle moving in resonance with wave characterized by the Klein-Gordon dispersion relation with cutoff or Compton frequency $\omega_{\mathrm{c}}=\mathrm{mc}^{2} / \hbar$. This approach brings field values (group velocity and intensity of transverse nonpropagating waves) into Maxwell's equations, and antiparticles or non-propagating waves with negative group velocity into Newtonian mechanics. In classical astrophysics, matter-antimatter interactions led to the universe expansion, formation of galaxiesand sources of intense cosmic radiation. Classical field theory could play a major role in clean energy research. In particular, concentrating solar power (CSP) systems, i.e. Stirling engine (SE) or small direct-heated tower, with thermal energy storage (TES), diffuse light-utilizing photovoltaic (PV) cell and 3D-printed thermal field emission (TFE) converters will dramatically lower the cost and energy payback time of the CSP technologies. The design explores recent developments in field-effect PV cells. 3D-printed TFE panels-integrated in TES or micro-channel solar receivers. The improved performance of the TFE converter compared to photovoltaic cells at high temperatures is attributed to the simultaneous use of diffusion and ballistic electron transport to harvest both thermal and photon energy. In addition, a gas cooled water moderated reactor is explored to integrate electrical-in-nature nuclear energy into smart micro-grids. and convert the most hazardous wastes into an emission-free fuel and medical isotopes. Transmutation reactor, in which depleted uranium acts initially as an absorber and later as fuel, behaves like fast reactors, but without their difficult control problems and high fissile fuel load.
\end{abstract}

Keywords: Klein-Gordon Equation, Non-propagating Waves, de Broglie Pilot Waves, 3D-printed Thermal Field Emission, Solar-to-electricity Converter, Gas-cooled Water Moderated Transmutation Reactor

\section{Introduction}

Several barriers made renewable energy less accessible. Solar energy has a variable power supply, and its energy content is both low and costly to store. Since the infrared part is not utilized to produce electricity, conventional PV cells use only about half of the solar energy. Direct radiation flux is necessary for concentrating solar power (CSP) to work efficiently. Outmost, the total abandonment of traditional space-time physics weakened the foundation of energy research. In particular, spent fuel and depleted uranium (DU) that could be a major source of nuclear energy and medical isotopes are now being managed as waste.

Classical physics greatly impacted the power industry by addressing the cross-disciplinary challenges of the environmentally-friendly nuclear and solar system design.

To increase reliability and reduce the cost of manufacture of solar power plants, Square Kilometer Array (SKA) with 3D-printed dishes and nightly operating Cherenkov Telescope Array (CTA) technologies can be utilized.

Positron annihilation spectroscopy can be used to study the dynamic vacuum effects, state and electron structure of the 
3D-printed thermal field emission (TFE) electrodes.

\section{Classical Field Theory}

Harmonic oscillators occupy an important place in classical physics, including construction of the Lorentz metrics representation and understanding the role of the phase. The Lorentz transformation relating the space and time coordinates of two reference systems, $\mathrm{K}$ and $\mathrm{K}^{\prime}$ in relative motion has been obtained as partial differential equations. From the natural properties of space and time: homogeneity, isotropy, etc., we have

$$
\begin{gathered}
d t^{\prime}=\frac{\partial t^{\prime}}{\partial t} d t+\left(\vec{V} \cdot g r a d t^{\prime}\right) d t=\frac{1}{\sqrt{1-V^{2} / c^{2}}}\left(d t-\frac{d \vec{r} \vec{V}}{c^{2}}\right) \\
d \vec{r}^{\prime}=\frac{1}{\sqrt{1-V^{2} / c^{2}}}\left(\frac{d \vec{r} \vec{V}}{V^{2}} \vec{V}-\vec{V} d t\right)+d \vec{r}-\frac{d \vec{r} \vec{V}}{V^{2}} \vec{V}
\end{gathered}
$$

Here, vector $\vec{V}(\vec{r}, t)$ is the velocity at a point fixed in reference system $\mathrm{K}^{\prime}$, and $\mathrm{c}$ is the constant. From the demand that $\mathrm{dt}^{\prime}$ in any kind of motion should be the total or convective derivative, we have

$$
\left.\frac{\partial \vec{V}}{\partial t}=-(\vec{V} \cdot \operatorname{grad}) \vec{V}\right)=\lambda(\vec{r}, t) \vec{V}
$$

Here $\lambda$ is an arbitrary function. As the total derivative of the velocity vector is equal to zero, the considered frames are inertial. We have that the function $t^{\prime}$ is a surface of constant phase and satisfies the eikonal or Hamilton-Jacobi equation of mechanics in dimensionless form

$$
c^{2}\left[\left(\frac{\partial t^{\prime}}{\partial x_{1}}\right)^{2}+\left(\frac{\partial t^{\prime}}{\partial x_{2}}\right)^{2}+\left(\frac{\partial t^{\prime}}{\partial x_{3}}\right)^{2}\right]-\left(\frac{\partial t^{\prime}}{\partial t}\right)^{2}+1=0
$$

This well-known duality exploited in theory of dispersive waves is based on the variation principle and Lagrange formalism. For any linear problem, it must be quadratic function of wave $\psi=\psi_{0} \mathrm{e}^{\mathrm{i} \theta}$ and its derivatives.

The average Lagrangian takes the form $\mathrm{L}=\mathrm{G} \times\left(\psi_{0}\right)^{2}$, where the dispersion relation $\mathrm{G}(\omega, \mathrm{k})$ is determined by the particular equations of the problem [1].

For linear partial equations, it is based on equivalence $\partial / \partial \mathrm{t} \leftrightarrow-\mathrm{i} \omega, \partial / \partial \mathrm{x} \leftrightarrow \mathrm{k}$, where $\mathrm{k}$ is a wave vector and $\omega$ is a frequency. For a wave associated with a free particle the elementary action is $\mathrm{ds}=\mathrm{hd} \theta=\mathrm{Ldt}$ and energy is $\mathrm{E}=\mathrm{pv}-\mathrm{L}$. In this case, the total energy is a constant of integration.

For a particle with a rest mass $m$ and linear momentum $p$, the total energy is $E^{2}=p^{2} c^{2}+m^{2} c^{4}$. For $v<<c$, the total energy is $E=p^{2} / 2 m$ and waves are described by the Schreodinger equation. These waves are described by the Klein-Gordon equation and dispersion relation $\omega^{2}=c^{2} k^{2}+\omega_{c}^{2}$, where $\omega_{c}$ is a constant called the cutoff frequency. The group velocity approaches zero and waves are not propagating if their frequency is below the cutoff frequency $\omega_{c}$.

Their group and phase velocity are related by $\mathrm{vu}=\mathrm{c}^{2}$, group velocity maximum is $\mathrm{c}$ and in the infinite $\mathrm{k}$ limit $\mathrm{v}=\mathrm{u}=\mathrm{c}$ [2].

A vibrating particle of the rest mass $m$ is moving with a wave characterized by the wave vector $\mathrm{k}=\mathrm{p} / \hbar$, frequency. $\omega=\mathrm{E} / \hbar$ and an exchange between rest-mass energy and wave energy at the cutoff or Compton frequency $\omega_{\mathrm{c}}=\mathrm{mc}^{2} / \hbar$, where $\hbar$ is Planck's constant, rest-mass energy $\mathrm{E}=\mathrm{mc}^{2}$ stabilizes the charged particles and $\mathrm{c}$ is the velocity of light.

Since dynamic vacuum is a dispersive medium, we can use the method of stationary phase developed for large times by Kelvin. The basic idea in Kelvin's approach is that the main contribution to the integral comes from the small intervals centered about those values or which the phases of the exponential are stationary. We have approximately

$$
K(x, t) \approx\left(t^{2}-x^{2} / c^{2}\right)^{-\frac{3}{4}} \cdot \exp \left(-l \frac{m c^{2}}{\hbar} \sqrt{t^{2}-x^{2} / c^{2}}\right)
$$

As in Feynman's method of path integral, $\mathrm{K}$ can be written as $\psi=\psi_{0} \mathrm{e}^{\mathrm{i} \theta}$. The amplitude, wave vector and frequency are no longer constant. The waves are interpreted in terms of the probability to found a particle at a given point.

\section{Wave Model of Long-range Forces}

In the case of a wave associated with a charged particle moving with the velocity $\vec{v}^{\prime}$ in the field created by the charged particles resting in the frame $\mathrm{K}^{\prime}$, we must add to the phase or elementary wave action $\mathrm{ds}^{\prime}=\omega_{\mathrm{c}} \mathrm{dt} t^{\prime}$ a supplementary term that described this interaction. It must be only scalar from the demand of the phase covariance. Then the wave action or charge phase pattern in the frame $\mathrm{K}^{\prime}$ is

$$
d s^{\prime}=-\left[m c^{2} \sqrt{1-v^{\prime 2} / c^{2}}+e \varphi\left(\vec{r}^{\prime}, t^{\prime}\right)\right] d t^{\prime} .
$$

Here the constant $\mathrm{e}$ is characterizing the charged particle and $\phi$ is an arbitrary function of the space-time coordinates. Introducing $\mathrm{dt}^{\prime}$ and $\mathrm{v}^{\prime}$ we obtain in the frame $\mathrm{K}$

$$
d s=\left[-m c^{2} \sqrt{1-v^{2} / c^{2}}+\frac{e}{c}(\vec{A} \vec{v})-e \phi(\vec{r}, t)\right] d t=L(\vec{r}, \vec{v}, t) d t .
$$

The wave equations describe electromagnetic phenomena in terms of potentials and the wave's group velocity, which plays a fundamental role.

A vector potential $\vec{A}=\frac{1}{c} \cdot \phi(\vec{r}, t) \cdot \vec{V}(\vec{r}, t)$ is proportional to the group velocity $\vec{V}(\vec{r}, t)$ and scalar potential

$$
\phi(\vec{r}, t)=\phi\left(\vec{r}^{\prime}, t^{\prime}\right) / \sqrt{1-V^{2} / c^{2}}
$$

From the Lagrange equation $\frac{d}{d t}(\partial L / \partial \vec{v})=\partial L / \partial \vec{r}$, we have 


$$
\frac{d \vec{p}}{d t}=-\frac{e}{c} \frac{\partial \vec{A}}{\partial t}-e \cdot \operatorname{grad} \varphi+\frac{e}{c}[\vec{v} \times \operatorname{curl} \vec{A}] .
$$

The first part of the Lorentz force in (7) is called the electric field intensity E. The second part is called the magnetic field intensity $\mathrm{H}$. It can be shown that the density of electromagnetic energy in vacuum is equal $\left(\mathrm{E}^{2}+\mathrm{H}^{2}\right) / 8 \pi$.

The electromagnetic fields remain invariant under the local gauge transformation $\vec{A}^{\prime}=\vec{A}-\nabla \psi$ and $\varphi^{\prime}=\varphi+\frac{1}{c} \frac{\partial \psi}{\partial t}$.

A common choice is $\operatorname{div} \vec{A}=0$ in empty space. Then $\vec{k}_{k}(t)=\vec{k} \vec{B}_{k}(t)=0$ and the electromagnetic waves are transverse waves. Introducing the partial derivative of vector $\vec{V}(\vec{r}, t)$ from (2), we can rewrite the equations for the field

$$
\begin{gathered}
\vec{E}=-\frac{1}{c^{2}} \partial \phi / \partial t \cdot \vec{V}-\frac{1}{c^{2}} \cdot \phi \cdot \lambda \cdot \vec{V}-\operatorname{grad} \phi \\
\vec{H}=\frac{1}{c} \cdot \phi \cdot \operatorname{curl} \vec{V}+1 / c[\operatorname{grad} \phi \times \vec{V}] .
\end{gathered}
$$

We multiply vectors of the electric field intensity and velocity,

$$
[\vec{V} \times \vec{E}]=[\operatorname{grad} \phi \times \vec{V}] \text { or } \vec{H}=\frac{1}{c}[\vec{V} \times \vec{E}] .
$$

Comparing with equation (8), we have

$$
\operatorname{curl} \vec{H}=\frac{1}{c}\{(\vec{E} \cdot \operatorname{grad}) \vec{V}-(\vec{V} \cdot \operatorname{grad}) \vec{E}+\vec{V} \cdot \operatorname{div} \vec{E}-\vec{E} \cdot \operatorname{div} \vec{V}\} .
$$

In continuum mechanics, some characteristics are conserved. From condition that the vector lines of the electric field intensity conserves in time (Helmgoltz's theorem of vortex line conservation

$$
\frac{d \vec{E}}{d t}-(\vec{E} \cdot \operatorname{grad}) \vec{V}+\vec{E} \cdot \operatorname{div} \vec{E}=0 \operatorname{curl} \vec{H}=\frac{1}{c}\left(\frac{\partial \vec{E}}{\partial t}+\vec{V} \cdot \operatorname{div} \vec{E}\right) .
$$

Taking the divergence of both sides of (9), we have $\partial \rho / \partial t+\operatorname{div}(\rho \cdot \vec{V})=0$, where we introduce a new function $\rho$. As it satisfies the continuity equation for wave connected with the particles created field, then $\rho$ is their charge density that is equal the charge $e_{0}$ multiplying on its probability amplitude $\psi^{2}$. From condition that the integral from function $\phi(r, t)$ by liquid volume conserves in time (the Lorentz condition) we derive the wave equations for the scalar and vector potentials [3].

The transformation from the Coulomb gauge-instantaneous potentials to the Lorenz gauge results in retarded solutions for scalar and vector potentials. Maxwell called $\varepsilon \mu \partial \mathrm{E} / \partial \mathrm{t}$ the displacement current Here vacuum permittivity $\varepsilon=36 \pi \mathrm{e}^{2} / \mathrm{hc}=8.8510^{-12} \mathrm{~F} / \mathrm{m}$, permeability $\mu=4 \pi 10^{-7} \mathrm{H} / \mathrm{m}$.

Light velocity $\mathrm{c}=1 /(\varepsilon \mu)^{1 / 2}=3 * 10^{8} \mathrm{~m} / \mathrm{s}$ is an invariant that can be derived in terms of particle-antiparticle pairs reacting like forced linear harmonic oscillators.

Since optical frequencies are much smaller than the cutoff frequency, the static limit of vacuum polarization are used to relate these constants to the properties of the vacuum.

The pairs such as electron-positrons described by the four Klein-Gordon equations are balanced by the energy $2 \mathrm{mc}^{2}$ at the distance $\mathrm{D}=\mathrm{e}^{2} /\left(4 \pi \varepsilon_{0} \mathrm{mc}^{2}\right)=1.4 \times 10^{-15} \mathrm{~m}$. Estimation of the electron's radiusis based on the ring shaped electron assumption.

The relative displacement equals the sum of the translation, deformation and rotation. This typical subdivision leads to two general classes of waves compressible and rotational.

As the transverse waves correspond to the electromagnetic field, it seems possible that longitudinal waves correspond to the gravitational field. By adding to the elementary action a supplementary term $-m \varphi\left(\vec{r}^{\prime}, t^{\prime}\right)$, we can compose the Lagrange function of a particle of mass $m$ moving with the velocity $\vec{v}^{\prime}$ in the wave field created by the large positive mass $\mathrm{M}$ resting in frame $\mathrm{K}^{\prime}$.

In steady-state non-rotational case $(\partial \mathrm{A} / \partial \mathrm{t}$ and $\operatorname{curl}(\vec{A})=0)$. We have the potential $\phi=-\mathrm{GM} / \mathrm{r}$ and the appearance of a constant $\mathrm{G}$ is a choice of units.

From the equation (3) in polar coordinates we have the law of planet orbital motion in the Sun field

$$
-\frac{1}{c^{2}}\left(\frac{\partial S}{\partial t}-\frac{G M}{r}\right)^{2}+\left(\frac{\partial S}{\partial r}\right)^{2}+\frac{1}{r^{2}}\left(\frac{\partial S}{\partial \phi}\right)^{2}+m^{2} c^{2}=0 .
$$

Antiparticles repulse each other gravitationally and tend to fill the entire cosmic space with homogenous density. The distinction in matter and antimatter gravitational acceleration led to the universe expansion, formation of galaxies and sources of intense cosmic radiation [4].

General relativity based on very complicated tensor formulations in curved space-time fails to explain the Cavendish experiment. The small spectral line shifts in enormous gravitational fields of dense stars challenge the principle of equivalence of gravity and acceleration.

Several additional hypotheses are required: space and time expansion; several exotic new particles; black holes; dark matter of an unknown kind and dark energy that is gravitationally repulsive.

Classical approach must play a major role in modern physics, given that the constancy of light velocity is restricted by algebra rules and field equations of general relativity do not lead to attraction between material bodies. The effects of vacuum fluctuation are confirmed in the Casimir attractive force between conductors, and Lamb spectral shift of the hydrogen atom. Here vacuum viewed as a spatially and temporally varying fluid prevents electrons to combine with nuclei in interior of atoms.

When the atom with atomic number $\mathrm{Z}$ and radius $\mathrm{R}$ is placed inside any electric field $\mathrm{E}$, there is a displacement of $\mathrm{x}$ between nucleus and electron cloud. At equilibrium the nucleus is balanced, so total effect of force must be zero and the induced electronic dipole moment $\mathrm{p}=\mathrm{zex}$.

At the electric field strength of about $5 \times 10^{5} \mathrm{~V} / \mathrm{m}$ and $\mathrm{R}_{\mathrm{a}}=10^{-10} \mathrm{~m}$, it can be shown that the displacement $\mathrm{x}$ of the hydrogen atom is about $10^{-16} \mathrm{~m}$. 


\section{Dynamic Vacuum Model}

The analogy between the non-propagating waves in medium and vacuum naturally appears when we consider waves on an infinite strip of width $h$. If $z(x, y, t)$ is the deflection of the strip, the solutions satisfying the boundary conditions $(\mathrm{z}=0$ or $\partial \mathrm{z} / \partial \mathrm{y}=0$ on the lines $\mathrm{y}=0$ and $\mathrm{y}=\mathrm{h})$ are normal modes $z=e^{\mathrm{iq}} \sin (\pi \mathrm{my} / \mathrm{h})$. Here $\mathrm{m}$ is the order of the mode and the $\mathrm{m}$-th cutoff frequency $\omega_{\mathrm{c}}=\pi \mathrm{mc} / \mathrm{h}$. A nondispersive mode corresponding to $\mathrm{m}=0$. As we approach wavelengths comparable with inter-atomic space, we consider a discrete string of atoms.

The frequency of the oscillation should be computed in terms of the mass of the atom $\mathrm{M}$ and the string elastic constant $\beta$ a. Here $a$ is the distance between two neighboring atoms, the length of the string of $\mathrm{N}$ atoms is $\mathrm{L}=\mathrm{Na}$, the material density $\rho=\mathrm{M} / \mathrm{a}$ and the characteristic speed $\mathrm{c}=(\beta \mathrm{a} / \rho)^{1 / 2}$.

It can be shown that deviation of the nth atom from the equilibrium position is $\mathrm{z}_{\mathrm{n}}=\mathrm{A}_{\mathrm{n}} \mathrm{e}^{\mathrm{i}(\omega t+\mathrm{kna})}$, where $\mathrm{n}$ is integer.

The internal energy density $u=2 \frac{\hbar c}{\pi^{3}} k_{m}^{2}$ and discrete set of $\mathrm{k}$ is connected with medium structure.

For wavelengths shorter than mean distance between the atoms $\left(\mathrm{k}_{\mathrm{m}}=\pi \mathrm{N} / \mathrm{L}=\pi / \mathrm{a}\right)$, propagation becomes impossible. As $\mathrm{k}_{\mathrm{m}}=10^{8} \mathrm{~cm}^{-1}$ and $\mathrm{c}=3 * 10^{5} \mathrm{~cm} / \mathrm{s}$, the maximum frequency of the material oscillations $\omega_{\mathrm{m}}=\mathrm{ck}_{\mathrm{m}} \approx 10^{13} \mathrm{~s}^{-1}$.

If the maximum frequencies of vacuum oscillations $v_{\mathrm{m}}$ is $\sim 10^{24} \mathrm{~s}^{-1}$, it leads to restriction on its characteristic size $>2 \mathrm{c} / v_{\mathrm{m}}=10^{-16} \mathrm{~m}$. Then the number of oscillators between $\mathrm{k}$ and $\mathrm{dk}$ in the polar coordinates is $d N=\frac{4 \pi}{(2 \pi)^{3}} k^{2} d k$ and their energy is $\hbar \mathrm{ck}$, we have $\mathrm{k}_{\mathrm{m}}=10^{16} \mathrm{~m}^{-1}$ and $u=3 \frac{\hbar c \pi}{(2 \pi)^{3}} k_{m}^{4} \approx 10^{37}$ $\mathrm{N} / \mathrm{m}^{2}$. The entire treatment is essentially the same for electromagnetic or nuclear matter. The unifying feature is the medium dispersion. Originally, Poincare introduced a scalar cohesive pressure that holds electrons together.

Electron was pictured as a sphere, in which energy $E=\mathrm{mc}^{2}$ compensates repulsive electrical forces. For ideal quantum fluid or radiation of energy density $\mathrm{u}$, the dynamic vacuum, pressure is $\mathrm{P}=\mathrm{u} / 3$. The vacuum pressure of $10^{36} \mathrm{~N} / \mathrm{m}^{2}$ or about $50,000 \mathrm{~N}$ strong force holds quarks together to form protons with size $\mathrm{R}_{0}=1.1 \times 10^{-15} \mathrm{~m}$.

A 1,000-N residual effect of this pressure holds the protons together to form the nucleus. Balance of nuclear and electromagnetic forces determines the structure of the nuclei.

Taking into account the Pauli Exclusion Principle, we have $d N=2 \frac{4 \pi}{(2 \pi)^{3}} k^{2} d k$.

All nuclei have essentially the same density, radii $\mathrm{R}=\mathrm{R}_{0} \mathrm{~A}^{1 / 3}$ and well potential energy $\mathrm{U}=\mathrm{E}_{\mathrm{F}}+\mathrm{E}_{\mathrm{b}}=45 \mathrm{MeV}$, where $\mathrm{k}_{\mathrm{F}}=1.52 / \mathrm{R}_{0}$ and $\mathrm{E}_{\mathrm{b}}=8 \mathrm{MeV}$.

In the pilot-wave model, the wave intensity and group velocity fields play role of hidden variables. Helmholtz equation is used to find the eigen-frequency modes in the atoms. It is assumed that dynamic vacuum pressure compensates Coulomb interactions in the atoms [5].

The physical aspect of an analytical model for Coulomb interactions between charged particles in high density beams requires the solution of space charge problems.

The paradox of experimental electrostatics is that similar charges have formed a cluster of terminal thickness near the conductor's surface. It is indirectly shown that vacuum pressure compensates Coulomb interactions in the intense electron beams [6].

\section{Classical Physics Approach in Clean Energy Research}

This section addresses to acceptance of classical theoretical approach in. environmentally sustainable electricity generation that is already affordable in remote areas with high energy costs. Electrical-in-nature nuclear energy could have an important role in the green energy effort, including countries that do not have fuel enrichment and reprocessing equipment. The novel CSP designs explore the astrophysical methods and experiments such as the Cherenkov Telescope Array (CTA) and Square Kilometer Array (SKA). In particular, 3D-printed SKA plastic antenna coated with conductive paint can serve as a prototype of from heliostat mirrors or dishes.

The Sandia National Laboratories (SNL) can provide unique capabilities, such as the National Solar Thermal Test Facility. It can be used to test novel technologies, i.e. fieldeffect ferroelectric PV cells and non-imaging secondary (NIS) mirror concepts.

In the recent CTA two-mirror antenna test, both the critical issues of the reutilization and possible adaptations of the telescopes to optimize them for light utilization from heliostat mirrors and would bring important performance improvements.

\subsection{Nuclear Energy Power Cycle}

To integrate electrical-in-nature nuclear energy into green micro-grids, a thermo-electric gas-cooled water moderated reactor with a TFE topping cycle can be used. In particular, a small modular reactor (SMR) could convert nuclear waste (depleted uranium (DU) and spent fuel) into an emission-free fuel and medical isotopes.

In nuclear thermal field emission (TFE) converters, fission fragments from the cathode fuel (i.e. unclad $\mathrm{U}-\mathrm{ZrC}$ or U$\mathrm{ZrH}_{\mathrm{x}}$ fuel) form plasma that acts as the gate to neutralize space charge.

They extract energy from high energy plasma electrons rather than low energy valence electrons in thermionic converters. The high pressure from the gap can be released into magneto-hydrodynamic (MHD) generators. A breedburn SMR with thermal energy storage (TES) integrated in shielding doesn't need to be refueled for about 15 years.

To utilize their nuclear decay energy, spent fuel elements 
in dry storage cask (DSC) can be used to convert $\mathrm{CO}_{2}$. into $\mathrm{CO}$ and $\mathrm{O}_{2}$ for further conversion $\mathrm{CO}$ into a useful fuel.

The core gain is approximately equal to $\mathrm{A} /\left(1-\mathrm{A} \times \mathrm{k}_{21}\right)$, where $\mathrm{k}_{11}, \mathrm{k}_{22}$, the multiplication factors of the zones 1 and 2 on their own; $\mathrm{k}_{21}$ and $\mathrm{k}_{12}$ are the coupling coefficients, and $\mathrm{A}=\mathrm{k}_{12} /\left(1-\mathrm{k}_{11}\right)\left(1-\mathrm{k}_{22}\right)$ is the gain of the core with $\mathrm{k}_{21}=0$.

A high reduction of nuclear waste can be realized in the near-critical $\Delta \mathrm{k}_{11}=10^{-3}$ or pulsed fast booster surrounded by the sub-critical blanket with a breeding ratio of 1.0 .

A neutron source with a tritium gas or plasma target from Phoenix Nuclear Labs $\left(\mathrm{Q}=5 \times 10^{14} \mathrm{n} / \mathrm{s}\right.$, beam power $\left.5 \mathrm{~kW}\right)$, and/or transfer of delayed neutron and gamma emitters from the blanket 22 can be used to control the booster 21 .

Average booster power $\mathrm{P}_{11}=\mathrm{Q} / \Delta \mathrm{k}_{11} \times 10^{17}=5 \mathrm{MW}$, peak or pulse power $100 \mathrm{MW}$ and frequency $\mathrm{f}=\left(\alpha \mathrm{P}_{11} / \mathrm{lS}\right)^{1 / 2}=50 \mathrm{~Hz}$ can be achieved. Here negative temperature feedback $\alpha=25^{*} 10^{6} /{ }^{\circ} \mathrm{C}$ and neutron lifetime $\mathrm{l}=10^{-7} \mathrm{~s}, \mathrm{P}_{11} / \mathrm{S}=10^{\circ} \mathrm{C} / \mathrm{s}$.
Heat losses from the coolant are minimized by the annulus gas which also enables leaks from the pressure tubes to be detected by any increases in humidity of the annulus gas.

The reactor, in which DU acts initially as an absorber and later as fuel, behaves like fast reactors, but without their difficult control problems and high fissile fuel load.

To achieve $70 \%$ efficiency, a TFE/MHD topping cycle reject heat to a $\mathrm{SCO}_{2}$ Brayton cycle, which in turn supplies heat to a Rankine cycle.

The core has feedback/vent loops 15 , steam generators 16 , charcoal-filled traps to delay passage of gas fission fragments with gas purification system, heat exchangers 17 and inner reflector 28 (layer of water backed by DU layer) [7-9].

Detectors 12 on the feedback loops or vent lines provide means for in-core monitoring and detection of a pressure tube leak (Figure 1).
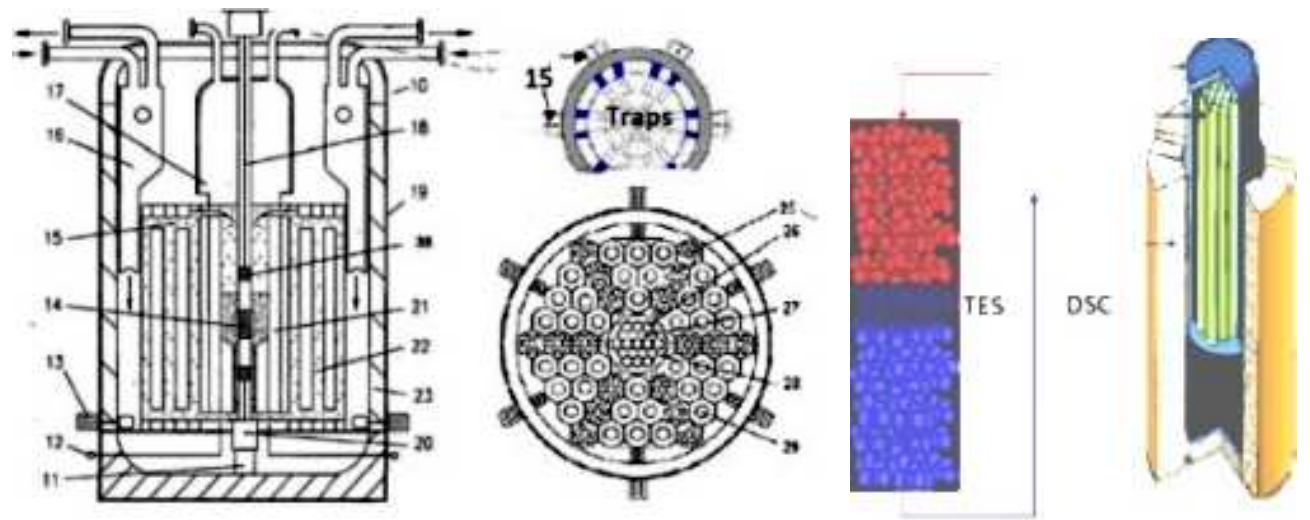

Figure 1. Breed and burn reactor, TES and DSC.

\subsection{Solar Energy Power Cycle}

The design of a concentrating solar power (CSP) systems with PV-assisted mirrors could be a first step towards collect the direct and diffuse components of the sunlight. The CSP with thermal field emission (TFE) converters as a topping cycle could have high overall efficiency. Heat from TFE anode is transferred to a bottom cycle. The design allows economical integration the TFE modules with a thermal storage. To meet the high efficiency need, TFE-CSP systems with a compressible fluid such as $\mathrm{CO}_{2}$, helium, or hydrogen at the optimum temperatures of $500-700^{\circ} \mathrm{C}$ are sought.

$3 \mathrm{D}$-printing reduces the manufacturing complexity, time and energy use. In the solar 3D-printed TFE converter, a trace made of a CNT ink can be printed on a dielectric substrate as an emitter, surrounded on both sides by a gate made of $\mathrm{Ag}$ or low work function $\mathrm{Ag}-\mathrm{O}-\mathrm{Cs}$ micro-particle ink.

Ag-O-Cs electrodes are highly sensitive to light, including near-infrared rays. In the double gates (high-k dielectric layer is used to facilitate pulse/AC mode operations. In the TFE modules, the movement of electrons between the emitter and the collector is controlled by the gate (grid). A gate electrode serving as an electron generator itself explores the enhancement of near-field thermal radiation to create on the cathode surface an electrostatic field large enough to compensate space charge field and initiates TFE process.

In double gate, including based on semiconductor technology, the closest to the emitter gate has a small negative bias voltage. The second gate is biased with a positive voltage, larger in magnitude than the deflector bias. The TFE can be run in DC or AC mode, particularly at the resonance frequency tuned to the transit time of electrons.

The current density of $10^{5} \mathrm{~A} / \mathrm{cm}^{2}$ close to the field emission limit was obtained from an electrically heated single horizontal carbon nano-tube (CNT).

The current density was $1.73 \mathrm{~mA} / \mathrm{cm}^{2}$ in CNT arrays at a bias voltage of $2.64 \mathrm{~V}$. At the electric field $5 \times 105 \mathrm{~V} / \mathrm{m}$ and turn on/off response times $150 / 500 \mathrm{~ns}$, it was a displacement current, rather than flow of charge [10].

Also, nano-grating comparable with the electron de Broglie wavelength may lead to effects similar to those created by doping with donors.

The resistivity values of Si-based nano-grating layers, for example, were approximately $10^{-2} \mathrm{Ohm} \mathrm{cm}$, similar to those of $\mathrm{Si}$ semiconductors doped with phosphorus impurities having a concentration of $1018 \mathrm{~cm}^{-3}$. Two 3D-printed TFE designs are proposed. One is a TFE converter with a 
transparent anode that can be used to thermally insulate the TES and harvest diffuse light that is lost in the traditional CSP. Also, a lower diurnal light intensity threshold exists, below which the traditional CSP will not operate.

A second near-field or photon-enhanced TFE design with a thin anode containing a large number of cooling microchannels can serve as the CSP receiver. A quartz tube window absorbs long wavelength emissions from the cavity, while transmitting the visible solar input power. The quartz bundle and honeycomb transparent windows significantly reduce cavity and open receiver convection losses [11-14].

The $\mathrm{CO}_{2}$ gas from the TFE anode/receiver at the temperatures of about $650^{\circ} \mathrm{C}$ heats $\mathrm{sCO}_{2}$ Brayton and then steam Rankine cycles.

A beam-down dish design that is based on the $25 \mathrm{kWe} \mathrm{SE}$ includes 5 - TES, 7 - cathode, 8 - anode, 10 - gate (Figure 2).
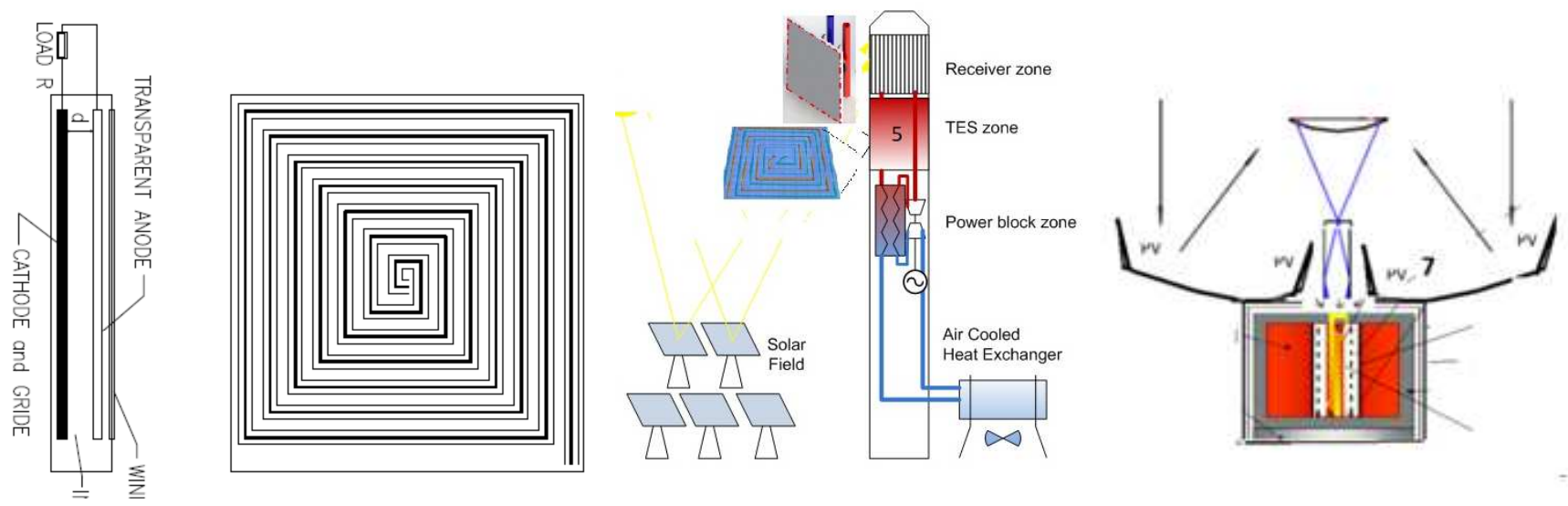

Figure 2. 3D-printed TFE, small tower plant and beam-down dish with TES.

\section{Results and Discussion}

Originally, the constancy of light velocity was postulated to justify the result of the Michelson experiment, instead of careful analysis of the systematic errors and despite it being met with opposition by many prominent physicists, including Michelson himself. Mathematically, it was based on the Lorentz transformation that preserves in moving frames a wave equation instead of time in the Galilean transformation.

As the same algebraic expression - Lorentz transformation - cannot give different results: constant light, but variable sound or any velocity, Einstein contradicts himself by using the variable values of light velocity in his original manuscript.

One of the non-relativistic ideas about the nature of reality at the quantum level is pilot wave theory proposed by de Broglie. In 1923, de Broglie suggested that a particle generates and interacts with some type of real waves and the wave interacts with the particle directing it.

Then Madelung provided a hydrodynamic-analog physical interpretation of the Schreodinger equation that yields a set of quantum Euler-like equations. Recently, the authors of work [5] considered a classical model of the hydrogen atom. The model consisted of the assumptions of a quantum fluid surrounding the proton. A density distribution of a dynamic quantum vacuum, and of a velocity field are describing the spatially varying speed of propagation of a disturbance in the density field.

In this dynamic system, acoustic-like resonances could be established describing the electron orbits as determined by the Helmholtz wave equation. When the wave theory leads to excellent agreement in all related experiments, there is disparity between the Michelson and Sagnac experiments, in which, anisotropy of light velocity is measured by sending the two signals in the opposite directions along the same path moving with velocity $\mathrm{v}=\Omega \mathrm{R}$.

In the Michelson experiment, $\Omega$ is angular velocity of the earth rotation around the sun and $\mathrm{R}$ is orbit radius. Unlike the Sagnac experiment on closed paths, signals are sending and detecting at two points in the Michelson open path experiment.

The result should be identical in these experiments, however, there is a zero-propagation time difference in the Michelson experiment. This is typical for systematic errors. In particular, the frequency change is proportional to propagation time change due to motion of the detectors.

The special theory of Relativity requires systems of clocksynchronized stationary observers and the constancy of light velocity based on the Lorentz transformation. Therefore, it is invalid due to its contradiction of both.

\section{Future Work}

To synchronize a grid with nuclear and solar power plants, the control method and heliostat tracking technology can utilize radio stars, particularly the sun, as a resilient frequency standard.

High speed photodiode and scalers can be used to analyze optical frequency difference in heliostat field for precise positioning of the beam on the receiver. To analyze the systematic effects in the Michelson experiment, a digital method was tested at Kiev Institute for Nuclear Research (KINR) [15].

The obtained experimental results could help with improving the accuracy of the wave model of a single-wire power transmission. In the sett-up to test the wave model, DC 
voltage was fed into the high-frequency generator with alternating current frequency of about $2.5 \mathrm{MHz}$, tuned to the resonant frequency of the open inductive-capacitive circuit.

The overall energy densities of conventional thermionic conversion are intrinsically low, because these systems are only able to free a few valence electrons. The energy density excited by fission fragments from the ground state of heavy ions, is about 105 times higher than energy densities of thermionic systems. As specific ionization losses in the low $\mathrm{Z}$ gas are much higher than in the high $\mathrm{Z}$ fuel, a gap of inert gas ( $5 \mathrm{~mm}$ at pressure $3 \mathrm{bar}$ ) has the total energy deposited by fission fragment is $75 \mathrm{MeV}$. Probes with electrically or radiation heated fission or calorimetric detectors can be used for in-core TFE test.

This includes optimum plasma electrical conductivity and energy deposition by secondary electrons and fission fragments. Overall dimensions of the calorimetric gamma detector (CGD) probe - diameter of $6.1 \mathrm{~mm}$ and length of 9.5 meters allow one to place it in the regular instrumentation channel's tube.

The SMR design can be based on gas jet and CANDU annulus gas systems. Fission fragment-ionized plasma from gaps between the fuel/cathode 7 and anode 8 is directed to MHD converter (Figure 3).
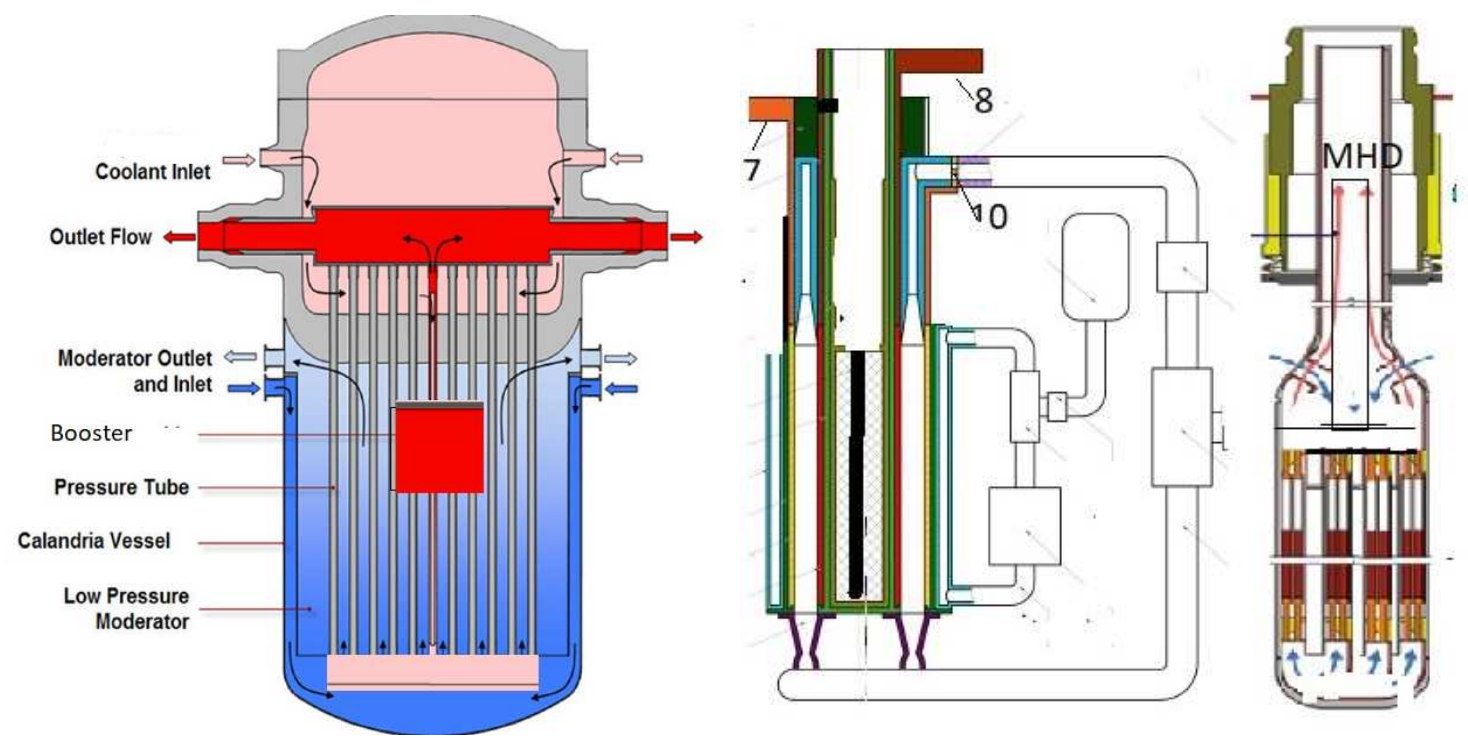

Figure 3. CANDUSMR, test loop and fuel channel.

Traditional space-time physics will be used in novel TFE converter design, including COMSOL or Monte Carlo modeling of innovative geometry and electromagnetic materials.

Recently, the experimental characterization of the fully 3D-printed carbon nano-tube (CNT) field emission electron sources was reported. The start-up voltages of about $60 \mathrm{~V}$ and $0.02 \mathrm{~cm}$-gap gate transmission $>97 \%$ were demonstrated [16].

Turn-on field can be decreased when emitter's temperature is increased in the proposed design. The incoming concentrated solar power is captured by a absorber plate of the receiver that is at $900-1,000^{\circ} \mathrm{C}$.

The absorber plate is in close proximity with the TFE, which converts about $25 \%$ of the thermal energy emitted by the absorber into electricity.

The remaining $75 \%$ is captured by a Thermal Energy Storage (TES) system, which can be used to run a heat engine with about $25 \%$ efficiency to produce electricity during off-sun hours. Total efficiency is $45 \%$.

The adjustable angle PV panels attached on periphery of the mirror or SE dish can be tested.

SE has maximum $33 \%$ efficiency and annual $\sim 23 \%$ efficiency to grid. The proposed solar converter would operate at high temperatures, enabling its waste heat to be used to power a secondary thermal engine.
Figure 4 illustrates the CSP with near-field TFE converter which has a $p$-doped narrow-bandgap semiconductor cathode separated from a gas-cooled anode with a vacuum gap. The unique feature of the near-field TFE is a sub-wavelength gap distance between the cathode and a heat source to allow nearfield radiation energy transfer between them.

This near-field thermal radiation can photo-excite a significant amount of electrons to the cathode conduction band if the cathode is made of a $p$-doped narrow-bandgap semiconductor, such as diamond film $(\mathrm{Eg}=1.54 \mathrm{eV}), \mathrm{InSb}$ $(\mathrm{E} g=0.17 \mathrm{eV}), \mathrm{GaSb}(\mathrm{E} g=0.726 \mathrm{eV})$ or InAs $(\mathrm{E} g=0.354 \mathrm{eV})$, where $\mathrm{E} g$ is the bandgap energy.

A photon-enhanced converter preferably used in the open receivers consists of a $p$-doped narrow-bandgap semiconductor as the absorber, a wide bandgap semiconductor as the barrier, and metal anode electrode.

From the perspective of thermionic emission, the absorber is treated as a cathode with a barrier of $\mathrm{Vc}$, whereas the electrode is treated as an anode, $\mathrm{d}$ is the absorber thickness [17].

As 3D-printed TFE converter material is similar to that used in photovoltaic cells, their performance at elevated temperatures is compared. The improved performance of the solid-state TFE converter at high temperatures is attributed to the simultaneous use of diffusion and ballistic transport of the electrons to harvest both thermal and photon energy. 
The National Solar Thermal Test Facility and $550^{\circ} \mathrm{C}$, Demonstration s- $\mathrm{CO}_{2}$ Brayton Power Conversion System can be used to test the TFE/receiver and non-imaging mirrors.
The high efficiency CSP system and solar TFE converter prototypes are shown in Figure 4.

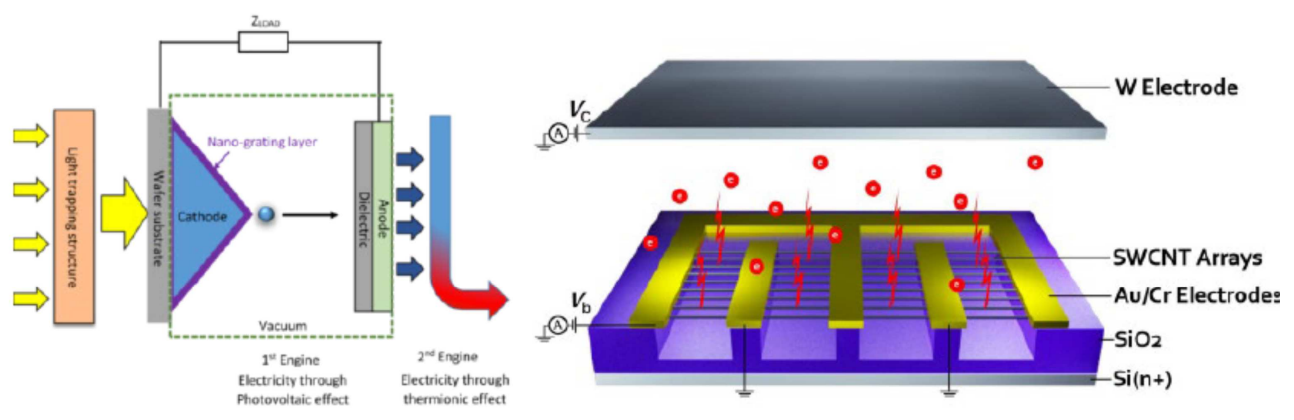

Figure 4. High efficiency CSP and TFE concepts.

\section{Conclusion}

Previous and planned experiments as well as a high theoretical efficiency $(70 \%)$ of the energy storage-integrated combined $\mathrm{sCO}_{2}$ Brayton and steam Rankine cycles confirm the viability of proposed modular nuclear and solar power plants to address global warming. A $100 \mathrm{MW}$ small modular reactor (SMR) with TFE/MHD converters as a topping cycle can be safely integrated into green micro-grid.

Current clean energy programs are incomplete without a long-term solution to nuclear waste problems. Since plutonium produces significantly less delayed neutrons than uranium, its use essentially compromises the safety of conventional fast reactors.

Due to a low inventory and fully integrated fuel cycle, the proposed reactor features exceptional sustainability and safety characteristics. Because of its flexibility, the design can achieve a wide range of conversion ratio from burner to breeder. The continuous transmutation of the actinides reduces the quality of the fissile plutonium inventory, making the SMR exceptionally proliferation resistant.

\section{References}

[1] A. Blanovsky, Classical Field Theory Beyond the Standard Model, Proceedings of the 22nd Workshop, Fundamental Problems of High Energy Physics and Field Theory, ed. by I. Filimonova and V. Petrov, Russia, pp. 165-177, (1999).

[2] S. Leibovich et al., Nonlinear waves, Cornell University Press, (1974).

[3] A. Blanovsky, Lorentz Electro-dynamics, Matter/Antimatter Cosmology and Astroparticle Physics, 7th International Wigner Symposium, College Park, MA, 24-29 (2001), (see http://arxiv.org/abs/physics/0401017).

[4] Y. Terletsky, Paradoxes in The Theory of Relativity. New York, NY: Plenum Press (1968).

[5] H. White et al. A discussion on a Dynamic Vacuum Model: Derivation of Helmholtz equation from Schrödinger equation, Physics Open, Vol. 1, 1000009, (2019).
[6] V. Sapogin, On Compensation of Coulomb Interaction of Charges by Beam's Self-consistent Field, Proceedings of the First International Conference on Circuits and Systems for Communications, Vol. 1, p. 408, (2002).

[7] A. Blanovsky, A Gas-cooled Water-moderated Thermoelectric Reactor Concept, Virtual Meeting, June 8-11, 2020, ANS Transactions, Vol. 122, p. 641 (2020).

[8] F. Jamerson, Investigations on the Direct Energy Conversion of Nuclear Fission Energy to Electrical Energy in a Plasma Diode, Nonr-3109(00), Office of Naval Research, (1967).

[9] A. Blanovsky, Model Based Breed-Burn Reactor, ANS Meeting, San Diego, CA, ANS Transactions, Vol. 102, p. 873 (2010).

[10] Y. Wang et al. On-Chip Thermionic Arrays Based on Horizontally Aligned CNT, EEE Transactions on Electron Devices, (2019).

[11] Y. Tsoglin and A. Blanovsky, Reliable In-core Power Monitoring System, ANS Winter Meeting, San Diego, ANS Transactions, Vol. 107, p 862 (2012).

[12] A. Blanovsky, Conceptual Design of Dielectric Accelerating Structures for Intense Neutron Sources, Advanced Accelerator Concepts Workshop, AIP Conference Proceedings, Volume 737; \#1, ed. by V. Yakimenko, New York, pp 295-301 (2004).

[13] N. Tauveronet al. Hybridization of a SMR with a Solar Power Plant Using a Supercritical Carbon Dioxide Brayton Cycle, 3rd European supercritical $\mathrm{CO}_{2}$ ConferenceSeptember Paris, France (2019).

[14] A. Blanovsky, Radioisotope Production Technology Demonstration Unit, ANS Winter Meeting, San Diego, Nov. 2012, ANS Transactions, Vol. 107, p. 97, (2012).

[15] A. Blanovsky, A New Method of Sagnac Effect Measurement on Long Baselines, KINR Reprint, (1989).

[16] Imperio Anel Perales-Martinez, and Luis Fernando VelásquezGarcía, Fully 3D-printed carbon nano-tube field emission electron sources within-plane gate electrode, Nanotechnology 30 495303, (2019).

[17] J. W. Schwede et al. Photon-enhanced thermionic emission for solar concentrator systems. Nat. Mater 9, 762-767, (2010). 